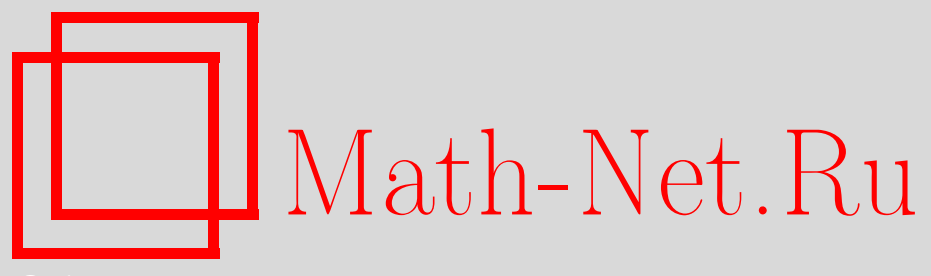

Т. У. Аубакиров, Е. Д. Нурсултанов, Пространства стохастических процессов, интерполяционные теоремы, УМH, 2006, том 61, выпуск 6, 181-182

DOI: https://doi.org/10.4213/rm5519

Использование Общероссийского математического портала Math-Net.Ru подразумевает, что вы прочитали и согласны с пользовательским соглашением http://www . mathnet.ru/rus/agreement

Параметры загрузки:

IP : 54.81 .137 .203

26 апреля 2023 г., 16:07:34

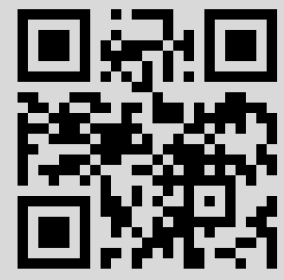




\section{Пространства стохастических процессов, интерполяционные теоремы}

\section{Т. У. Аубакиров, Е. Д. Нурсултанов}

В работе вводятся пространства стохастических процессов, которые являются аналогами сетевых пространств [1]. Доказываются интерполяционные свойства и теоремы вложения.

Будем предполагать заданным полное вероятностное пространство $(\Omega, \mathscr{F}, \mathrm{P})$ с фильтрацией, т.е. семейством $\mathbf{F}=\left\{F_{n}\right\}_{n \geqslant 1} \sigma$-алгебр $F_{n}$ таких, что $F_{1} \subseteq \cdots \subseteq F_{n} \subseteq \cdots \subseteq \mathscr{F}$.

Для заданного стохастического процесса $X=\left(X_{n}, F_{n}\right)_{n \geqslant 1}$ и $k \in \mathbb{N}$ обозначим

$$
\bar{X}_{k}=\sup _{A \in F_{k}, \mathrm{P}(A)>0} \frac{1}{\mathrm{P}(A)}\left|\int_{A} X_{k} \mathrm{P}(d \omega)\right| .
$$

Через $N_{p q}(\mathbf{F}), 0<p<\infty, 0<q \leqslant \infty$, обозначим множество стохастических процессов $X=\left(X_{n}, F_{n}\right)_{n \geqslant 1}$, для которых при $q<\infty$

$$
\|X\|_{N_{p q}(\mathbf{F})}^{q}:=\sum_{k=1}^{\infty} k^{-1-\frac{q}{p}} \bar{X}_{k}^{q}<\infty
$$

и при $q=\infty$

$$
\|X\|_{N_{p \infty}(\mathbf{F})}:=\sup _{k} k^{-\frac{1}{p}} \bar{X}_{k}<\infty .
$$

$N_{p q}(\mathbf{F})$ будет квазинормированным пространством (при $q \geqslant 1$ нормированным) как факторпространство по ядру

$$
J=\left\{X: \int_{A} X_{k} \mathrm{P}(d \omega)=0, A \in F_{k}, k \geqslant 1\right\} .
$$

Введенные пространства $N_{p q}(\mathbf{F})$ характеризуют усиленный закон больших чисел для стохастических процессов. Если $X \in N_{p q}(\mathbf{F})$, то последовательность $\left\{X_{k}(\omega) / k\right\}_{k=1}^{\infty}$ почти всюду стремится к нулю таким образом, что

$$
\left.\sum_{k=1}^{\infty}\left(k^{\frac{1}{p^{\prime}}} \frac{\left(X_{k}(\omega)\right.}{k}\right)\right)^{q} \frac{1}{k}<\infty .
$$

Пусть стохастический процесс $X=\left(X_{n}, F_{n}\right)_{n \geqslant 1}$ является субмартингалом. Тогда

1) при $0<q_{1} \leqslant q \leqslant \infty$ верно $\|X\|_{N_{p q}(\mathbf{F})} \leqslant c\|X\|_{N_{p q_{1}}(\mathbf{F})}$;

2) при $0<p<p_{1}<\infty, 0<q, q_{1} \leqslant \infty$ верно $\|X\|_{N_{p q}(\mathbf{F})} \leqslant c\|X\|_{N_{p_{1} q_{1}}(\mathbf{F})}$.

Пусть $T=\left\{T_{n}\right\}_{n=1}^{\infty}-$ преобразование стохастических процессов, определенных на $(\Omega, \mathscr{F}, \mu)$ с фильтрацией $\mathbf{F}=\left\{F_{n}\right\}$, в стохастические процессы, определенные на $(\Lambda, \mathscr{R}, \nu)$ с фильтрацией $\boldsymbol{\Phi}=\left\{\Phi_{n}\right\}: T(X)=\left\{T_{n}(X), \Phi_{n}\right\}_{n=1}^{\infty}$. Будем говорить, что преобразование $T$ квазилинейно, если найдется $c>0$ такое, что для любого $n \in \mathbb{N}$ верно $\overline{T_{n}(X)-T_{n}(Y)} \leqslant c \overline{T_{n}(X-Y)}$.

Пусть $X=\left(X_{n}, F_{n}\right)_{n \geqslant 1}-$ стохастический процесс, $\tau(\omega)$ - марковский момент [2]. Через $X^{\tau}$ обозначим “остановленный" процесс $X^{\tau}=\left(X_{n \wedge \tau}, F_{n}\right)_{n \geqslant 1}$, где $X_{n \wedge \tau}=\sum_{m=1}^{n-1} X_{m} \chi_{\tau=m}(\omega)+X_{n} \chi_{\tau \geqslant n}(\omega)$, а через $X^{*}-$ стохастический процесс $\left(\max _{1 \leqslant k \leqslant n} X_{k}, F_{n}\right)_{n \geqslant 1}$.

Данные преобразования стохастического процесса $X$ являются примерами квазилинейных преобразований.

Tеорема 1. Пусть $0<p_{0}<p_{1}<\infty, 0<r_{0}<r_{1}<\infty, 0<\theta<1,1 \leqslant s \leqslant \infty$, $\frac{1}{r}=\frac{1-\theta}{r_{0}}+\frac{\theta}{r_{1}}, \frac{1}{p}=\frac{1-\theta}{p_{0}}+\frac{\theta}{p_{1}}, X=\left(X_{n}, F_{n}\right)_{n \geqslant 1}-$ субмартингал $и T=\left\{T_{n}\right\}_{n=1}^{\infty}-$ квазилинейное преобразование. Если для любого $k \in \mathbb{N}$ выполнены условия

$$
\left\|T\left(X^{k}\right)\right\|_{N_{r_{1} \infty}(\mathbf{\Phi})} \leqslant M_{0}\left\|X^{k}\right\|_{N_{p_{1} 1}(\mathbf{F})}, \quad\left\|T\left(X-X^{k}\right)\right\|_{N_{r_{0} \infty}(\mathbf{\Phi})} \leqslant M_{1}\left\|X-X^{k}\right\|_{N_{p_{0} 1}(\mathbf{F})},
$$


mo

$$
\|T(X)\|_{N_{r s}(\mathbf{\Phi})} \leqslant c \max \left(M_{0}, M_{1}\right)\|X\|_{N_{p s}(\mathbf{F})} .
$$

Данная теорема отличается от классических интерполяционных теорем [3]. Здесь интерполируются не пространства, а преобразование фиксированного стохастического процесса, т.е. условия и утверждение теоремы относятся к одному процессу.

Теорема 2. Пусть процесс $X=\left(X_{n}, F_{n}\right)_{n \geqslant 1}$ является субмартингалом, $\tau(\omega)$ марковский момент. Тогда имеют место неравенства

$$
\left\|X^{\tau}\right\|_{N_{p q}(\mathbf{F})} \leqslant c\|X\|_{N_{p q}(\mathbf{F})}, \quad\left\|X^{*}\right\|_{N_{p q}(\mathbf{F})} \leqslant c\|X\|_{N_{p q}(\mathbf{F})} .
$$

Пусть $1<p<\infty, p^{\prime}=p /(p-1), 1<q \leqslant \infty, \alpha \in \mathbb{R}$. Определим пространство мартингалов

$$
N_{p}^{\alpha q}(\mathbf{F})=\left\{X=\left(X_{n}, F_{n}\right)_{n \geqslant 1}-\text { мартингал : } \sum_{k=0}^{\infty}\left(2^{\alpha k} \overline{\Delta X_{k}}\right)^{q}<\infty\right\},
$$

где

$$
\overline{\Delta X_{k}}=\sup _{A \in \mathscr{F}, \mathrm{P}(A)>0} \frac{1}{[\mathrm{P}(A)]^{\frac{1}{p^{\prime}}}}\left|\int_{A}\left(X_{2^{k+1}}-X_{2^{k}}\right) P(d \omega)\right|, \quad X_{2^{-1}} \equiv 0 .
$$

$N_{p}^{\alpha q}(\mathbf{F})$ является пространством сходящихся мартингалов, где параметры $\alpha, q$ и $p$ характеризуют скорость и метрику, в которой сходится данный процесс.

Пусть $\mathbf{A}=\left(A_{0}(\mathbf{F}), A_{1}(\mathbf{F})\right)$ - пара квазинормированных собственных подпространств линейного хаусдорфова пространства $\mathscr{N}(\mathbf{F})$ стохастических процессов, определенных на вероятностном пространстве $(\Omega, \mathscr{F}, \mathrm{P})$ с фильтрацией $\mathbf{F}=\left\{F_{n}\right\}_{n \geqslant 1}$.

Для $0<\theta<1$ при $0<q<\infty$ обозначим

$$
\left(A_{0}, A_{1}\right)_{\theta, q}=\left\{X \in \mathscr{N}(\mathbf{F}):\|X\|_{\left(A_{0}, A_{1}\right)_{\theta, q}}^{q}=\int_{0}^{\infty}\left(t^{-\theta} K(t, X)\right)^{q} \frac{d t}{t}<\infty\right\},
$$

а при $q=\infty$

$$
\left(A_{0}, A_{1}\right)_{\theta, \infty}=\left\{X \in \mathscr{N}(\mathbf{F}):\|X\|_{\left(A_{0}, A_{1}\right)_{\theta, \infty}}=\sup _{0<t<\infty} t^{-\theta} K(t, X)<\infty\right\},
$$

где $K\left(t, X ; A_{0}, A_{1}\right)=\inf _{X=Y+Z}\left(\|Y\|_{A_{0}}+t\|Z\|_{A_{1}}\right)$ - функционал Петре [3].

Tеорема 3. Пусть $1<p<\infty, 1 \leqslant q, q_{0}, q_{1} \leqslant \infty, \alpha_{0}<\alpha_{1}, 0<\theta<1, \alpha=$ $(1-\theta) \alpha_{0}+\theta \alpha_{1}$. Тогда $\left(N_{p}^{\alpha_{0} q_{0}}(\mathbf{F}), N_{p}^{\alpha_{1} q_{1}}(\mathbf{F})\right)_{\theta, q}=N_{p}^{\alpha q}(\mathbf{F})$.

Теорема 4. Пусть фильтрация $\mathbf{F}=\left\{F_{n}\right\}_{n \geqslant 1}$ такова, что для каждого $k=$ $1,2, \ldots$ и любого $A \in F_{k}$ выполнено условие $\mathrm{P}(A) \geqslant \frac{c}{k}$, где постолнная $c>0$ не зависит от $k$. Если $1<r<p<\infty, 1 \leqslant q \leqslant \infty, \alpha=\frac{1}{r}-\frac{1}{p}$, то имеет место вложение $N_{r}^{\alpha q}(\mathbf{F}) \hookrightarrow N_{p q}(\mathbf{F})$.

\section{Список литературы}

[1] Е. Д. Нурсултанов, Матем.сб., 189:3 (1998), 83-102. [2] А. Н. Ширяев, Вероятность, Наука, М., 2005. [3] Й. Берг, Й. Лефстрем, Интерполяиионные пространства. Введение, Мир, М., 1980.

\section{T. У. Аубакиров (T. U. Aubakirov)}

Казахстанский филиал

Московского государственного университета

им. М. В. Ломоносова

\section{Е. Д. Нурсултанов (E. D. Nursultanov)}

Казахстанский филиал

Московского государственного университета

им. М. В. Ломоносова

E-mail: er-nurs@yandex.ru
Представлено А. Г. Костюченко Принято редколлегией 08.09.2006 